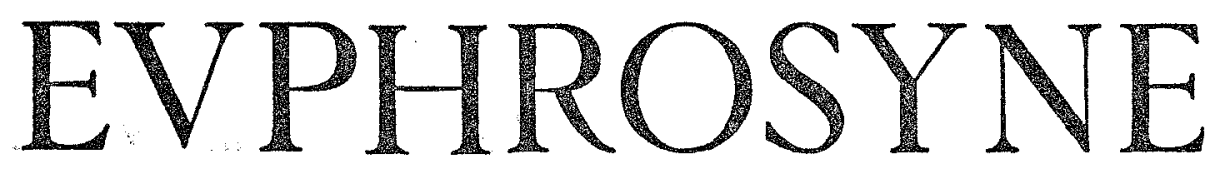

\title{
REVISTA DE FILOLOGIA CLÁSSICA
}

NOVA SERIE-VOLUME XXIII

SEPARATA

JESUS-MARÍA NIETO
IBANEZ
Condicionamientos sintácticos
en la desaparición del infinitivo
en griego antiguo

LISBOA - 1995 


\title{
Condicionamientos sintácticos en la desaparición del infinitivo en griego antiguo ${ }^{1}$
}

\author{
JESÚS-MARÍA NIETO \\ IBÁÑEZ \\ Universidad de León (España)
}

En su evolución al griego medieval y moderno todo el sistema verbal griego experimenta una transformación muy profunda y marcada. Como es bien sabido, de los modos personales desaparece el optativo y de los no personales el infinitivo, mientras que el participio es sustituido por un gerundio indeclinable. Por otra parte, dentro de los temas verbales han surgido nuevas formas perifrásticas sustitutivas de los antiguos futuros y perfectos ${ }^{2}$.

Y quizá uno de los aspectos más llamativos y curiosos de la sintaxis del griego tardío sea precisamente la desaparición del infinitivo. Resulta realmente sorprendente que una forma verbal que conoció un uso tan intenso y extenso en el griego arcaico y clásico sea totalmente sustituida y eliminada. En efecto, los únicos restos que quedan del infinitivo en el griego moderno son formas totalmente fosilizadas, auténticos sustantivos del tipo tò $\varphi \alpha \gamma^{\prime}$

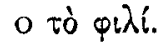

Sin embargo, al igual que en los demás fenómenos lingüísticos, el cambio no se ha producido de un modo espontáneo e instantáneo, sino que el

1 Agradezco al profesor Emilio Crespo las interesantes observaciones realizadas sobre la versión manuscrita anterior a este artículo. No obstante, he de decir que todas las omisiones e incorrecciones que queden en él son de mi entera responsabilidad.

2 Para una visión general de los cambios sintácticos de esta época es fundamental el trabajo de M. García Teijeiro, «Innovaciones sintácticas en la koiné», Actas del VI Congreso Español de Estudios Clásicos, Madrid, 1983, pp. 247-278. 
proceso se iba gestando en la lengua desde estadios anteriores. En la evolución del griego la koiné ${ }^{3}$ es una etapa clave; en esta época se producen importantes cambios en todo el sistema lingüistico griego, cambios que ahora aparecen por escrito, pero que ya se habían gestado en las etapas anteriores de la lengua. Desde este momento hay que contar con una disociación entre la lengua escrita y la lengua hablada, que, aún sin llegar a ser diglosia, sin embargo era bastante acentuada. En este punto no hay que perder de vista el hecho de que apenas contamos con testimonios directos de la lengua hablada, ya que todo documento escrito, tanto popular como oficial, no escapa a la tradición literaria anterior, por el convencionalismo y conservadurismo propio de la escritura, y pasará aún un tiempo antes de que los griegos escriban tal y como hablan. Conscientes de este problema, para ilustrar este artículo en el período de la koiné nos serviremos básicamente de ejemplos tomados de los papiros y del Nuevo Testamento, dado que éstos nos proporcionan un conocimiento más directo de la lengua hablada del momento, y por lo tanto un más fiel reflejo de los usos sintácticos del infinitivo en esta etapa de la evolución.

En los textos de este período la sintaxis del infinitivo muestra una serie de desviaciones con respecto a la normativa clásica que preludian ya su total desaparición posterior. La sintaxis del infinitivo testimonia perfectamente este conflicto y compromiso entre un sistema desusado, que es la norma escrita, y un sistema vivo, que es el habla viva del momento, cuyo reflejo más claro es la sustitución del infinitivo por construcciones con conjunción y forma personal, y por el refuerzo mediante el artículo, conjunciones y otras partículas. Por ello, la historia del infínitivo en este período parece a primera vista contradictoria: por una parte pierde cada vez más terreno al ser desplazado por oraciones subordinadas, y por otra aumenta grandemente el uso del infinitivo sustantivado; lo primero es un fenómeno de la lengua popular, lo segundo una particularidad del estilo literario ${ }^{4}$.

Este cruce de los dos niveles de lengua hace que la gramática de la koiné sea una gramática de faltas o errores, pero lo que es una falta a juicio de la sintaxis normativa aparece ya como el florecimiento de un nuevo sistema.

Muchos son los motivos que han incidido en este proceso de sustitución o, mejor dicho, de eliminación del infintivo, ya que los elementos que entran en juego son de múltiple naturaleza: fonéticos, morfológicos, sintácticos, semánticos, etc. Evidentemente hay que partir de la evolución fonética del sistema vocálico de la koiné, fundamentalmente del itacismo y de la pérdida

3 Para el problema de la cronología de este período lingüistico vid. L. Gil, «Ojeada a la koiné: ensayo de caracterización periodológica», Minerva, 1, 1987, 81-91.

4 O. Hoffmann, A. Debrunner y A. Scherer, Historia de la lengua griega, trad. esp., Madrid, 1973, pp. 344 y ss. 
de la noción de cantidad. La reducción del diptongo /ei/a /i/ y de /ai/ a /e/ ${ }^{5}$ daba lugar a confusiones entre las diferentes formaciones del infinitivo. En la koiné aparecen ya por escrito todas estas alteraciones fonético-morfológicas. F. T. Gignac ${ }^{6}$ recoge formaciones de infinitivo con una terminación en $-\varepsilon \iota$, en $-\iota$ y alguna en $-\varepsilon v$, además de - $\varepsilon \iota v$. Por otra parte, hay un acercamiento fónico entre el infinitivo de presente y el del aoristo, lo que provoca acciones reguladoras e intercambios de desinencias entre el infinitivo de presente y el infinitivo de aoristo ${ }^{7}$. En la voz pasiva se observa la misma tendencia, la desinencia - $\sigma \theta \alpha$, con la reducción del diptongo /ai/, se confunde con la desinencia personal $-\sigma \theta \varepsilon$. Todo ello, junto con la eliminación atemática (- $\nu \alpha \iota$ y $-\varepsilon v \alpha \iota)$, hizo que el infinitivo, como tal categoría verbal, no contase con un apoyo formal. Además, hay que tener presente que la reducción de los grupos - $105,-10 \mathrm{~V}$ a $-15,-t(v)$ respectivamente desempeñó un importante papel en la desaparición del infinitivo ${ }^{8}$. Las terminaciones fonéticas del infinitivo en $-\imath \nu$ o $-\imath$ se asimilan formalmente a este tipo de sustantivos homófonos, preludio de los neutros en $-\imath$ del griego moderno. Es el caso de

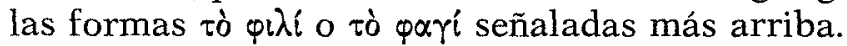

Desaparecida así la oposición fonética entre las diferentes formas, el sistema de los modos y de los tiempos, se desestabiliza, por lo cual la lengua creará otras nuevas formas de expresión a partir de otros procedimientos lingüisticos que los refuercen y caractericen. En el caso concreto del infinitivo nos encontramos con el empleo de refuerzos y apoyos formales, como son el artículo o las conjunciones. Los valores semánticos del infinitivo no desaparecen con él, sino que son asumidos por otras construcciones según veremos a lo largo de este artículo.

Ahora bien, estas transformaciones fonéticas no han venido sino a dinamizar y a dar el impulso definitivo a un proceso que ya se venía gestando en el seno de la lengua griega. El infinitivo, un sistema totalmente estable, a primera vista, es sustituido por un nuevo sistema sin relación aparente con el anterior. Entre ambos polos hay un período de desestabilización en el que confluyen elementos de ambos sistemas lingüisticos con hipercorrecciones y cruces de diferentes niveles. Precisamente, este trabajo se va a centrar en esta etapa lingüistica con el objeto de mostrar que el elemento fundamental de esta desestabilización no parece ser la transformación foné-

5 Tradicionalmente se han venido situando estos cambios fonéticos a partir del siglo II a. C., si bien los estudios de S. T. Teodorsson, The Phonemic System of the Attic Dialect 400-340 a. C., Lund, 1974, y I. R. Alfageme, "Notas sobre la evolución del sistema vocálico en la koiné», CFC, $9,1975,339-379$, ponen de manifiesto que estos fenómenos de confusiones gráficas y fonéticas son anteriores, al menos en algunas capas de la población.

6 A Grammar of the Greek Papyri of the Roman and Byzantine periods. II Morphology, Milano, 1981, pp. 330 y ss.

7 Gignac, op. cit., pp. 346 y ss.

8 Gignac, op. cit., pp. 25 y ss. 
tica, como ocurre en gran parte de los cambios del griego de la koiné, sino su propia sintaxis ${ }^{9}$. En efecto, el problema del infinitivo es diferente, ya que no presentaba una sintaxis estable, según lo prueban diversos testimonios, algunos bastante anteriores a este período. Ahora bien, en esta etapa aumentan considerablemente estos casos de debilitamiento del infinitivo debido a la mayor presencia de elementos de la lengua hablada en la lengua escrita. El infinitivo muestra, en este sentido, una situación y evolución diferente a la del subjuntivo, que es un ejemplo claro de mantenimiento de una categoría verbal contra la que no ha podido la evolución fonética de la lengua. El subjuntivo no podía desaparecer, sus funciones estaban perfectamente definidas y delimitadas en el contexto sintáctico por oposición al indicativo, hecho que no ocurría con el infinitivo.

La desaparición del infinitivo griego tiene sus raíces mucho más atrás, y viene marcada por una tendencia general a reforzar formalmente el infinitivo. Ya desde sus propios orígenes el infinitivo llevaba en sí el germen de la inestabilidad, debido a su propia naturaleza nominal y verbal ${ }^{10}$. La combinación de esta doble naturaleza da lugar a unos usos por una parte propios de un sustantivo, $y$, por otra, propios del verbo, que a veces son contradictorios, lo que hace que resulte muy complejo sistematizar toda la variedad de usos sintácticos del infinitivo griego originados de esta naturaleza peculiar.

Como decían los propios griegos, el infinitivo es $\alpha \dot{\pi} \alpha \rho \rho^{\mu} \mu \rho \alpha \tau o \zeta, ~ « f o r m a ~ q u e$ no poesee significación accesoria». En efecto, el infinitivo es un tema nominal que expresa la idea verbal pura y simple, abstraída de todo modo y persona, lo que hace que la libertad de empleo del infinitivo sea muy grande, sin caer en la definición excesivamente general de $P$. Burguière ${ }^{11}$, según la cual todos los múltiples valores del infinitivo pueden resumirse en una función de explicitación de un contexto verbal o nominal, como desarrollo y determinación de un elemento de la frase. Aunque sí podemos hablar de una cierta «ambigüedad», o, más bien, de una falta de marcas formales en cada contexto que precisen el valor que tiene en él, y por ello esta amplitud de valores va a ser delimitada y caracterizada por la propia lengua griega, de acuerdo con el contexto nominal o verbal en que aparezca el infinitivo.

9 Así ocurre, por ejemplo, en la evolución del futuro y de los modos verbales, según demuestran los trabajos de J. M. Floristán Imízcoz para el caso de Epicteto, como reflejo de la lengua popular de la koiné imperial, «El tema de futuro en Epicteto», EClás, 89, 1986, 111-131, y «Los modos verbales en Epicteto", Minerva, 1, 1987, 93-106.

10 Sobre el origen nominal del infinitivo puede consultarse para el caso del indoeuropeo A. Meillet, "Les cases employés à l'infinitif en indo-européen»,BSL, 32, 1931, 188-193, y E. Benveniste, Origenes de la formation des noms en indo-européen, Paris, 1936, pp. 129-134, y para el caso concreto del griego E. Schwyzer y A. Debrunner, Griechische Grammatik, vol. II, München, 1950 , pp. 358 y ss, y J. Haudry, «Hypothèses sur l'origine des infinitifs en grec ancien", $B S L, 70,1975,115-136$.

11 Histoire de l'infinitif en grec, Paris, 1960, p. 31. 
De ahí que esta capacidad del infinitivo para asumir una serie de funciones poco o nada diferenciadas desde el punto de vista morfológico, aunque sí mediante otros procedimientos, ha llevado a la lengua a crear diversos refuerzos o expedientes formales para precisar mejor su valor. Este proceso se inició ya desde los propios orígenes del infinitivo, si bien su mayor extensión se da en los textos de la koiné. Veámoslo:

\section{El empleo del artículo para indicar las diferentes funciones casuales.}

Como es bien sabido, en todas las épocas de la lengua griega el infinitivo puede, a pesar de la ausencia de flexión y del artículo, asociarse y desarrollar diversas funciones sintácticas. Sin embargo, un cambio decisivo para la historia del infinitivo se produjo con la incorporación del artículo a sus usos, pues éste evita la ambigüedad de las diferentes funciones casuales. En este punto, siguiendo a L. Gil ${ }^{12}$, debemos calibrar en la justa medida la profunda alteración que supuso para el sistema del infinitivo griego su sustantivación, que yendo en progresión creciente terminó por provocar su ruina y convertirlo en un simple sustantivo.

En el griego helenístico aumenta considerablemente el uso del infinitivo sustantivado. En los papiros estos infinitivos son típicos del estilo oficial, aunque no faltan en textos populares ${ }^{13}$. En el Nuevo Testamento se hallan, sobre todo, en los autores más cultos, como San Lucas o San Pablo, según se desprende de los datos estadísticos de Aalto ${ }^{14}$ para esta época. Como caracterizan también a la literatura de la época, especialmente a la obra de Polibio ${ }^{15}$, está claro que su extensión en la koiné se debe a una particularidad de estilo literario tendente a un mayor empleo de nombres abstractos ${ }^{16}$. Sin embargo, el verdadero motor que hace aumentar su empleo en esta época es esa tendencia a reforzar y dar una cierta iden-

12 L. Gil, «Sobre el origen y evolución del genitivo del infinitivo articular griego con valor final», Emerita, 21, 1953, 48-58, concretamente p. 58.

13 Vid. ejemplos en B. G. Mandilaras, The Verb in the Greek non-literary Papyri, Athens, 1973, pp. 331-351, y E. mayser, Grammatik der griechischen Papyri aus der Ptolemäerzeit, vol. II 1, Berlin-Leipzig, 1926 (reimpr. 1970), pp. 320-339.

14 Studien zur Geschichte des Infinitivus im Griechischen, Helsinki, 1953, pp. 65 y ss. Ejemplos de infinitivo articular en el Nuevo Testamento pueden verse en F. Balss y A. Debrunner, A Greek Grammar of the New Testament and other Early Christian Literature, Chicago, $1967^{9}$, pp. 205-212, y J. H. Moulton, A Grammar of the New Testament Greek, vol. III, Edinburgh, 1963 (reimpr. 1980), pp. 140-149.

15 E. G. W. Hewlett, «On the articular Infinitive in Polybius», AJPh, 11, 1980, 267-290 y $440-470$.

16 El mayor desarrollo del infinitivo articular tiene lugar a partir del siglo $\mathrm{V}$ y es más frecuente en la prosa culta debido al mayor empleo de nombres abstractos, según las estadísticas de B. L. Gildersleeve, "Contributions to the history of the articular Infinitive", TAPhA, 34, $1878,5-19$. Cfr. también J. Vendryés, "L'infinitif substantivé dans la langue de Lysias", $R P h$, $70,1954,113-133$. 
tidad al infinitivo en un momento en que está siendo sustituido por otras expresiones.

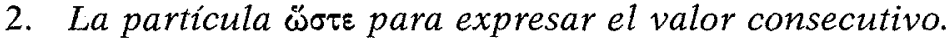

En un principio la consecuencia se expresaba sólo con el infinitivo, mientras que el empleo de un verbo finito es de origen tardío, ya que no aparece hasta época clásica ${ }^{17}$. Según se desprende del uso homérico, en esta época coexisten formas de infinitivo con un valor consecutivo con y sin la partí-

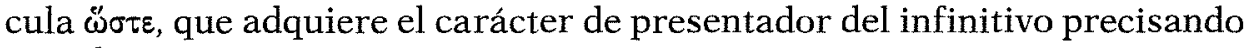
su valor consecutivo ${ }^{18}$.

\section{El presentativo toũ con valores propios, sobre todo de finalidad.}

Sin duda el sintagma privilegiado de la flexión del infinitivo articular es su empleo con el artículo en infinitivo. Desde que aparece por primera vez en Esquilo ha sido utilizado para expresar múltiples circunstancias, en muchos casos imprecisas, que a veces tenemos que traducir por una perífrasis, "por lo que respecta a», "cuando se trata de ...». Con el paso del tiempo sus usos se hacen tan diversos y cada vez más mecánicos que se pierde el sentido originario de relación causal del genitivo, convirtiéndose, en el griego de la koiné, el genitivo del artículo en un mero presentador del infinitivo para poner de relieve su carácter nominal.

En este período son numerosos los ejemplos donde se da la presencia de toũ delante del infinitivo en funciones no específicas de genitivo:

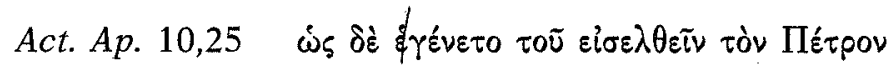

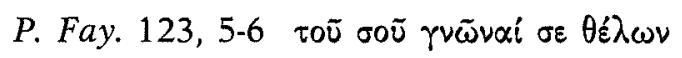

Sin embargo, en autores anteriores se detectan ya precedentes de este fenómeno:

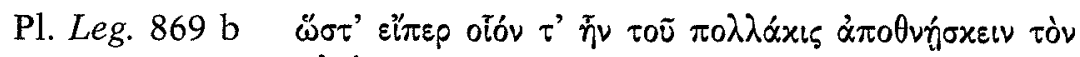

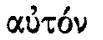

Quizá el caso más conocido sea su empleo con valor final ${ }^{19}$, atestiguado desde Tucídides. En este caso el roũ funciona a modo de presentador del infi-

17 Burguière, op. cit., p. 75.

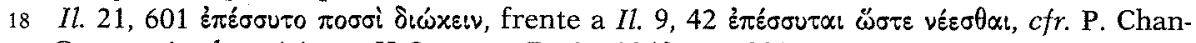
traine, Grammaire homérique. II Syntaxe, Paris, 1963, pp. 321 y ss.

19 Los filólogos no se ponen de acuerdo sobre su origen. Frente a A. Ernout ("L'infinitif grec et gérondif latin», $R P h, 71,1945,93-115)$, para quien se trata de un simple uso antiguo

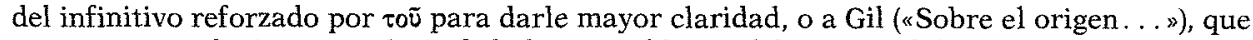
ve aquí una evolución secundaria de la función ablativa del genitivo del infinitivo articular que se ha desligado por completo del nexo que le unía con una determinada palabra de la frase, 
nitivo, casi como una conjunción. Para su alta frecuencia en la Septuaginta y en el Nuevo Testamento ${ }^{20}$, tanto con verbos de movimiento como de otro tipo, se ha intentado buscar una influencia semítica ${ }^{21}$, si bien parece, a juicio de Burguière ${ }^{22}$, un fenómeno estilístico. En los mismos Evangelios alterna la construcción de infinitivo con y sin toũ con el mismo sentido de finalidad, siguiendo en el primer caso el uso arcaico del infinitivo solo para explicitar un elemento de la frase. Tal es el caso de la conocida frase bíblica:

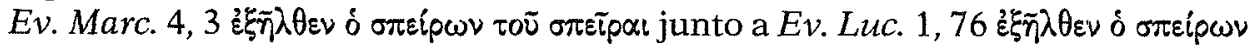

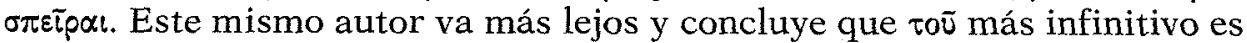
un hipercultismo, un refinamiento de estilo para expresar la finalidad, en una época en que la construcción de ǐv más subjuntivo, generalizada por la lengua hablada, está asumiendo la mayoría de las funciones del infinitivo.

Está claro que en estos ejemplos toũ está petrificado, ha perdido todo su valor casual y se ha convertido en una mera partícula de refuerzo del infinitivo, marcando así la conexión con el contexto en un momento en que está en proceso de desaparición.

En el período tardío se extiende considerablemente el empleo de estos refuerzos, de una manera artificial en muchos casos, para salvar el infinitivo seriamente amenazado por el avance de las construcciones conjuntivas. Y, precisamente, es éste otro de los elementos dinamizadores de la eliminación del infinitivo. La preferencia de la lengua por la claridad de las construcciones analíticas ha hecho que poco a poco el infinitivo haya ido perdiendo terreno ante ellas.

En efecto, en el período tardío las oraciones subordinadas van desplazando a todas las construcciones del infinitivo, con ö $\tau \iota$ en el caso de las oraciones declarativas, y con ǐvo más subjuntivo para los demás tipos de subordinada. Son numerosos los ejemplos donde aparece una construcción subordinada con conjunción en las condiciones en que en clásico aparecería el infinitivo solo:

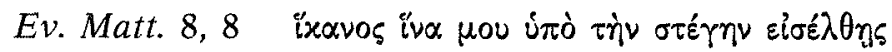

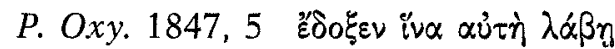

Esta situación llega al extremo con las oraciones completivas, donde el infinitivo empieza a sufrir antes la competencia de las conjunciones. Los pre-

\footnotetext{
está la opinión de Burguière (op. cit., pp. 132 y ss) que lo considera como un valor puramente accidental en la historia de este infinitivo. Para este autor el origen hay que buscarlo en la flexibilidad de los empleos del genitivo, y en la diversidad de relaciones que es capaz de expresar. Este giro tiende a asumir diversos matices circunstanciales, muy poco precisos, y poco a poco se va desligando del contexto, especializándose en un valor final en un gran número de ejemplos.

20 Según Mayser, op. cit., pp. 321 y ss, en los Papiros Ptolemaicos no hay ejemplos claros.

21 Vid., por ejemplo, H. J. Thackeray, A Grammar of the Old Testament in Greek according to the Septuaginta, Cambridge, 1909, p. 24.

22 Op. cit., pp. 138-139.
} 
cedentes de este resultado hay que buscarlos en una serie de alteraciones presentes ya en autores clásicos. En el texto de Heródoto, Tucídides o Jenofonte, por ejemplo, encontramos casos de alternancia sintáctica entre la construcción con infinitivo y los giros con conjunción:

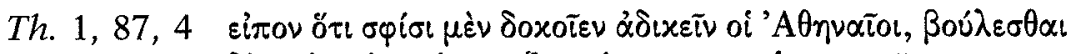

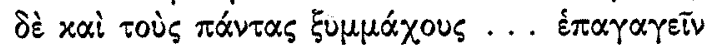

Es un ejemplo claro de equivalencia sintáctica, que revela la atracción que ejerce una construcción sobre la otra, y el grado de fragilidad de una de ellas.

Lo mismo indican los casos de contaminación de ambas construcciones que aparecen en los Papiros Ptolemaicos, el Nuevo Testamento, y que también se detectan en autores anteriores:

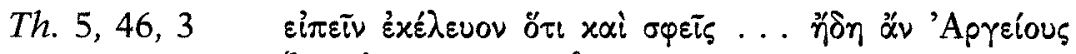

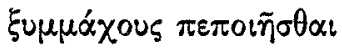

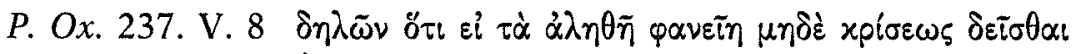
$\tau \grave{o} \pi \rho \tilde{\alpha} \gamma \mu \alpha$

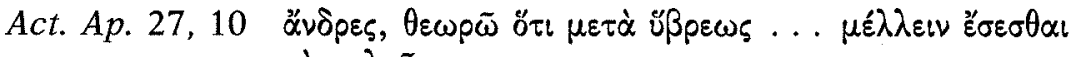
tòv $\pi \lambda$ oũv

En época tardía la construcción con conjunción va eclipsando al infinitivo, de forma que nos la vamos encontrar en verbos que en ático regían completiva con infinitivo:

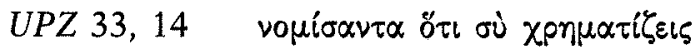

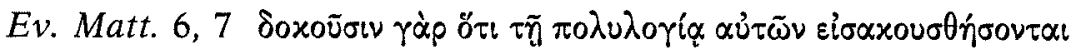

Todos estos fenómenos revelan un conflicto entre hechos de lengua de un nivel muy diferente, entre el conservadurismo propio de la tradición escrita, fiel a la construcción con infinitivo, y el impulso de la lengua hablada que opta por la claridad de la subordinación.

En esta sustitución del infinitivo por construcciones con conjunción y forma personal hay que tener presente también el problema de la expresión del aspecto verbal. Como ya hemos mencionado al comienzo de este artículo, la evolución fonética del griego tardío iba borrando las diferencias formales entre el infinitivo de presente y el de aoristo, lo que imposibilitaba al infinitivo expresar valores aspectuales, y temporales en el caso del futuro. La lengua griega, en cambio, tanto la medieval como la moderna, sigue manteniendo como fundamental la oposición aspectual entre el presente y el aoristo ${ }^{23}$, de forma que es totalmente imprescindible la sustitución del infi-

23 Cfr. A. Mirambel, «Essai sur l'évolution du verbe en grec byzantin», BSL, 61, 1966 167-190, y R. Browning, Medieval and modern Greek, London, 1969, pp. 36 y ss. 
nitivo por otras alternativas que expresen plenamente las categorías verbales, incluido el aspecto.

Sin embargo, en este proceso de desaparición hay un punto fundamental que es complementario de los anteriores. El infinitivo, por expresar la idea verbal pura y simple, es el término neutro, el término no marcado de las oposiciones sintácticas en que aparece. El infinitivo como tal no indica modo, es indiferente a ésta y a otras nociones, ya que no pertenece ni al modo indicativo, ni al potencial, ni al irreal, ni al imperativo, y precisamente por su valor neutro o indiferente puede aparecer en sustitución de cualquiera de ellos ${ }^{24}$.

Desde época antigua el infinitivo es utilizado para expresar una orden o un deseo ${ }^{25}$. En época tardía este uso está muy atestiguado en los documentos oficiales, edictos y cartas privadas ${ }^{26}$, si bien aparecen ya casos donde se yuxtapone el imperativo y el infinitivo en una misma frase, evidenciando la artificialidad de la construcción:

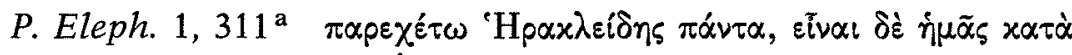 toutó}

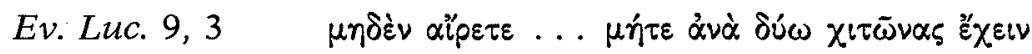

En estos casos el infinitivo es sustituido por una forma más marcada y precisa para manifestar una orden, como es el imperativo.

Las oraciones consecutivas introducidas por $̋ \sigma \tau \varepsilon$ presentan en griego una doble posibilidad de construcción: con modo personal o con infinitivo. Tradicionalmente estamos acostumbrados a considerar como real la consecuencia expresada por los modos personales, y como posible la expresada por el infinitivo. Sin embargo, el infinitivo puede aparecer en lugar del modo personal esperado ${ }^{27}$, precisamente por ser el término no marcado de esta oposición ${ }^{28}$ y no indicar modo ${ }^{29}$.

24 Cfr. E. Crespo, "Infinitivo modal sin ö้v en griego antiguo», EClás, 87, 1984, 67-73, y, sobre todo, J. L. García Ramón, «Los modos en las subordinadas consecutivas en griego clásico", Actas del VII Congreso Español de Estudios Clásicos, Madrid, 1987, pp. 155-161.

25 En las inscripciones dialectales es muy frecuente este infinitivo con valor prescriptivo, incluso por encima del propio imperativo, sobre todo en las inscripciones más antiguas, cfr. C. D. Buck, The Greek Dialects, Chicago, 1965, p. 140.

26 Vid. ejemplos de los papiros en Mandilaras, op. cit., pp. 316-320, Mayser, op. cit., pp. 303-305, y del Nuevo Testamento en Blass-Debrunner, op. cit., pp. 196-197, y Moulton, op. cit., p. 78.

27 E. Schwyzer y A. Debrunner, Griechische Grammatik. II Syntax und syntatische Stilistik, München, 1950, pp. 677-678.

28 Cfr. H. Kurzová, Zur syntaktischen Struktur des Griechischen. Infinitiv und Nebensatz, Amsterdam, 1968.

29 Cfr. García Ramón, op. cit. 
Incluso esta idea es válida para explicar el retroceso del infinitivo en las oraciones completivas. Desde los primeros testimonios escritos nos encontramos en griego con la coexistencia de la construcción con infinitivo y la construcción con conjunción en las oraciones completivas. Sin embargo, las dos construcciones no presentan una distribución indistinta y equivalente ${ }^{30}$ : admiten infinitivo o conjunción los verba sentiendi, sciendi y dicendi, mientras que sólo admiten infinitivo los verba volendi, imperandi y putandi. Está claro, entonces, que el infinitivo es compatible con cualquier tipo de verbos, ya que neutraliza el modo y la modalidad de la frase ${ }^{31}$. Como ya hemos visto, ante esta situación la lengua ha ido poco a poco sustituyendo, para acabar eliminándola, la forma neutra de complementación, que es el infinitivo, por una forma más marcada y precisa como es la subordinación mediante la conjunción y el modo personal. En definitiva, este carácter neutro del infinitivo junto con su capacidad para expresar cualquier tipo de determinación ha llevado a su propia ruina y sustitución por otros procedimientos marcados y más precisos con los que ya contaba la lengua.

Es evidente, entonces, que la sustitución, o mejor dicho, la eliminación del infinitivo no es un hecho repentino o puntual, sino que desde su mismo origen el infinitivo era inestable, a causa de su doble naturaleza verbal y nominal, de su deficiencia para la plena expresión de las categorias verbales, de su carácter neutro y de su capacidad para expresar diversas funciones sintácticas sin necesidad de una marca morfológica ni de un signo de relación con el contexto ${ }^{32}$. Y evidentemente no podemos olvidar la evolución fonética de la koiné helenística que ha contribuido de una forma decisiva y definitiva a acelerar este proceso.

Por todo lo que hemos dicho, el infinitivo tenía la batalla perdida ante el avance de las construcciones subordinadas. En concreto el giro de iv $\alpha$ más subjuntivo va a ser el triunfador en este proceso.

"Iv $\alpha$ se vacía de todo su valor y deja de ser una conjunción final para

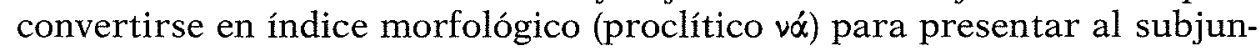

30 Para el problema de la justificación de la doble posibilidad de construcción puede consultarse la obra citada de Kurzová, y para un enfoque más reciente las obras de D. Lighfoot, Natural logic and the Greek Moods, The Hague-Paris, 1975, de G. de Boel, «Towards a Theory of the Meaning of complementizers in Classical Attic», Lingua, 52, 1980, 285-304.

$31 C f r$. E. Crespo, "On the System of substantive clauses in ancient Greek: a functional approach», Glotta, 62, 1984, 1-16, y J. de la Villa, "La subordinación completiva en función de sujeto en griego", Habis, 18-19, 1987-8, 9-27.

32 A este respecto H. Kurzová («Zum Aussterben des Infinitivs im Griechischen», Estudios Balcánicos Checoslovacos, 1, 1966, 39-50, 1966,y «Zur Problem des Infinitivschwundes in Griechischen», $L F, 92,1969,24-27)$ comparando esta situación con algunas lenguas balcánicas donde también el infinitivo es sustituido por los mismos elementos formales, concluye que el retroceso y posterior abandono de esta forma verbal se debe a la propia estructura de las construcciones sintácticas que permitía. 
tivo en un momento en que las diferencias entre el subjuntivo y el indicativo habían sido eliminadas por la fonética.

Para justificar el empleo de ívo más subjuntivo en lugar del infinitivo se ha de proceder con una perspectiva histórica. Desde Homero está atestiguado el empleo de subjuntivo acompañado de iv $\alpha$ después de verbos de "esfuerzo, súplica, voluntad, orden, etc.», verbos que también se acomodan al infinitivo, ya que éste por sí mismo es capaz de explicitar y completar cualquier idea verbal. He aquí algún ejemplo de esta situación:

frente a

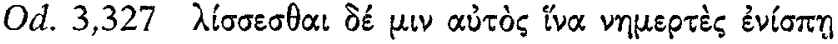

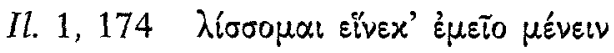

Existía pues una doble posibilidad en la lengua griega para prolongar el significado de un verbo, lo que permitirá y explicará la extensión de una de las dos alternativas en época tardía.

El triunfo de esta construcción analítica se debe a una serie de ventajas que no tenía la construcción sintética del infinitivo: expresa la persona, el sujeto está en nominativo, no en acusativo, y además materializa la función de dependencia a través de un elemento constante, superando así la ambi-

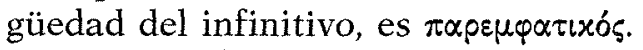

A pesar de todo, tardará aún un tiempo en imponerse este nuevo procedimiento, y habrá que pasar por diversas etapas de confluencia de ambas formas de expresión, como lo demuestra, por ejemplo, el texto de $1 \mathrm{Ep}$. Co. 15,5 , donde en una misma frase aparecen las dos construcciones, el infinitivo y íva más subjuntivo, preludio ya del giro que va a sustituir al infinitivo

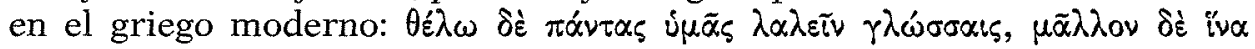

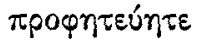

En la koiné estamos asistiendo a un proceso de refección general de un sistema, donde el infinitivo tenía inicialmente un lugar destacado. Este proceso de sustitución por el subjuntivo da lugar a conflictos y compromisos entre ambas construcciones, fruto del choque entre la lengua popular hablada y la culta escrita. A ello se deben una serie de contaminaciones anacolúticas que se detectan en los testimonios escritos de esta época:

1. Los casos ya comentados de infinitivo tras conjunción completiva.

2. Lo más importante son los constantes cruces que se producen entre el dominio del subjuntivo y del infinitivo:

- Los papiros ofrecen bastantes ejemplos de infinitivos tras ö $\pi \omega \varsigma$ o iva, en lugar del subjuntivo esperado:

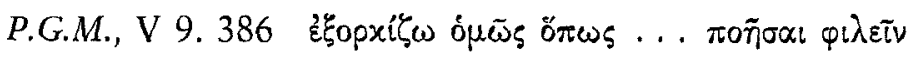

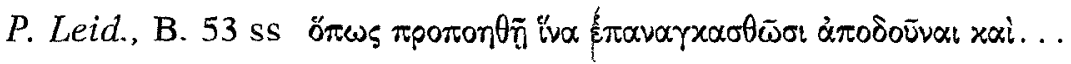




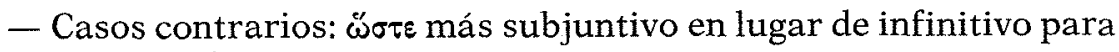
expresar la consecuencia:

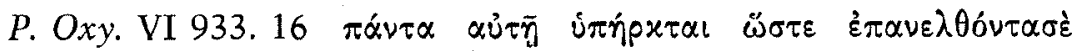

$$
\begin{aligned}
& \mu \alpha \rho \tau \text { นp } \eta \tilde{n}
\end{aligned}
$$

- Subjuntivo tras locuciones preposicionales con artículo para indicar diversas circunstancias:

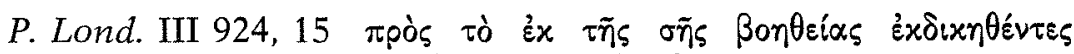 $\delta \cup v 0 \theta \bar{\omega} \mu \varepsilon \nu \ldots . \dot{\varepsilon} \times \tau \varepsilon \lambda \varepsilon \bar{\varepsilon} \nu$}

En éste y en casos similares, la influencia de un estilo escrito mantenido artificialmente no ha podido vencer a la costumbre de la lengua hablada que en las oraciones circunstanciales no conoce otro modo que el subjuntivo.

Estas irregularidades no son sino hipercorrecciones e hipercultismos en un momento de reajuste del sistema, que demuestran que el infinitivo ya no era algo vivo, sino algo propio del lenguaje literario y culto mantenido artificialmente por la escritura.

Como hemos podido comprobar, ya desde siglos antes de su desaparición se detectan aparentes irregularidades a la norma sintáctica del infinitivo en autores calificados de modélicos o clásicos ${ }^{33}$, que indican una nueva modalidad en la expresión sintáctica, tendente a la eliminación del infinitivo, que no pudo penetrar plenamente en la lengua escrita hasta que las circunstancias socio-culturales se lo permitieron, es decir, hasta la koiné.

\section{SUMMARY}

In the koiné the syntax of the infinitive shows a series of differences as regards classical standars (on the one hand the replacement of the infinitive by conjunctive constructions and verbal personal forms, and on the other, its reinforcement by means or articles, conjunctions and other particles) which anticipate its later complete disappearance. However, the main reason for this loss of stabilization in the infinitive does not seem to be the typical phonetic change that takes place at this stage of the language, but its own syntax, that is, the infinitive as the neuter term, the unmarked element of those syntactic oppositions in which it is found.

33 Quizá el caso más llamativo sea el de Tucídides; en dos conocidos artículos ( Del ático a la koiné», Emerita, 49, 1981, 377-392, y «Tucídides y la koiné», Athlon, vol. I, Madrid, 1989, pp. 245-261) A. López Eire observa algunas coincidencias entre la lengua de Tucídides y la koiné, lo que le lleva a afirmar que la koiné no es más que una nueva lengua conversacional que surge en Atenas en un momento en que ésta se convierte en capital de un imperio, y Tucídides es un autor que se ha dejado influir por el nuevo ático surgido en el nivel conversacional. 